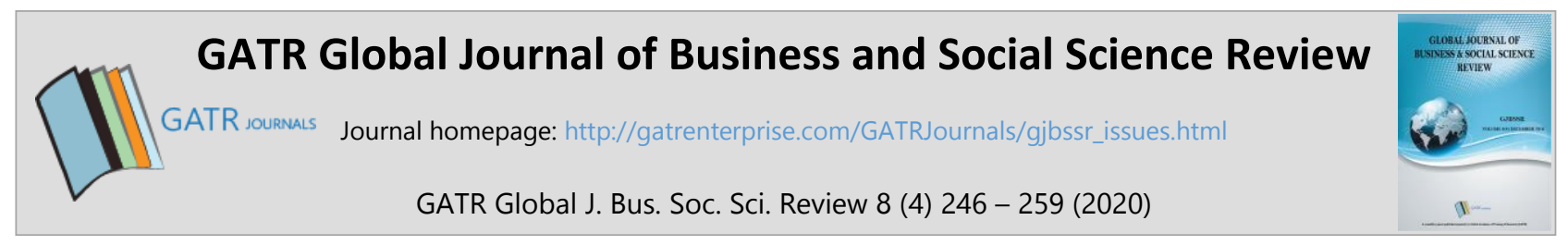

\title{
Brain Electrical Activity Mapping (BEAM) on Trait Anxiety among Malaysian Chinese Children
}

\section{Chen Chen Cheong $^{1 *}$, Asmidawati Ashari ${ }^{1}$, Rahimah Ibrahim ${ }^{1}$, Wan Aliaa W. Sulaiman ${ }^{2}$, Koo Kian Yong ${ }^{3}$}

${ }^{1}$ Department of Human Development and Family Studies, Faculty of Human Ecology, University Putra Malaysia, 43400 UPM, Selangor, Malaysia.

${ }^{2}$ Department of Medicine, Faculty of Medicine and Health Sciences, University Putra Malaysia, 43400 UPM, Selangor, Malaysia.

${ }^{3}$ New Mind Brain Health Centre, Wisma Life Care, No.5, Jalan Kerinchi, 59200 Bangsar South, Kuala Lumpur, Malaysia.

\begin{abstract}
Objective - Woefully, the early onset of anxiety disorders had affected children in different aspects throughout their developmental stages. In order to get rid of the increased prevalence rate among children, the biological attributed risk factors for anxiety should be given more concern. Particularly, this research intended to study the biological brain mechanism for trait anxiety among children. With brain electrical activity mapping, this research was aimed to study the relationship between the brain locations situated at the prefrontal cortex and temporal lobe with trait anxiety. Subsequently, this research aimed to predict the associated brain locations for trait anxiety among anxious children.

Methodology/Technique - A total of 212 Chinese children from Kuala Lumpur, Malaysia with high trait anxiety was recruited after the first phase of the screening phase through the administration of the State-Trait Anxiety Inventory for Children-Trait Scale (STAIC-T). Recruited children then proceeded to the second phase of brain electrical activity brain mapping with a Quantitative Electroencephalogram (qEEG) brain mapping machine.

Finding - Results showed that brain locations Fp1, Fp2, F7, F8, F3, F4, T3, and T4 are significantly correlated with trait anxiety while F8, Fp2, F4, and Fp1 are the significant predictors for trait anxiety among children during on task state. In short, the biological brain mechanism of brain locations played a role in forming the anxious trait the personality of children which resulted in reducing their resilience towards stress.

Type of Paper: Empirical

JEL Classification: D83, I19

Keywords: Brain Electrical Activity Mapping (BEAM); Children; Chinese; Malaysia; Trait anxiety; Quantitative Electroencephalogram (qEEG)

Reference to this paper should be made as follows: Cheong, C.C; Ashari, A; Ibrahim, R; Sulaiman, W.A.W; Yong, K.K. (2020). Brain Electrical Activity Mapping (BEAM) on Trait Anxiety among Malaysian Chinese Children, GATR Global J. Bus. Soc. Sci. Review, 8(4): 246 - 259. https://doi.org/10.35609/gjbssr.2020.8.4(6)
\end{abstract}

\section{Introduction}

Woefully, the twenty-first century is described as an era of anxiety (Malcolm, 2015; Karas, 2013). In fact, the increasing prevalence rate of anxiety disorder has afflicted children at alarming rates nowadays.

\footnotetext{
${ }^{*}$ Paper Info: Revised: September 30, 2020

Accepted: December 31, 2020

* Corresponding author: Chen Chen Cheong

E-mail: ccchen0810@hotmail.com;gs52156@student.upm.edu.my

Affiliation: Faculty of Human Ecology, University Putra Malaysia, Malaysia.
} 
However, early onset of childhood anxiety is still underestimated. Consistent and intense feeling of fear had affected them in various aspects like emotions, behaviours and cognitive functions throughout their developmental stage (Bittner et al., 2007; Pine, Cohen, Gurley, Brook, \& Ma, 1998; Woodward \& Fergusson, 2001). Without proper early intervention, children are at risk for anxiety disorders with more severe anxiety symptoms when they are growing up. In order to get rid of the elevating of prevalence rate for anxiety, understanding the etiology of the onset of anxiety should be given more concern. The Biopsychosocial Model of anxiety demonstrated that the etiology of anxiety disorders is the result from the interplay of these three aspects (Engel, 1997). In fact, this Biopsychosocial model views anxiety disorders as the products of biological aspects (gene and brain mechanism), psychological aspect (beliefs, behavior, coping skills) and social aspect (environmental factor). Among these three aspects, biological attributed vulnerabilities formed the first anxious personality in children since birth and reduced their resilience towards psychological and social stressors later on in their life (Averill, 2015).

In order to study the biological aspect of anxiety, the characteristics of trait anxiety make it a good indicator to do so. For instance, trait anxiety is the long-term anxious feeling that suffered by that individual that is hard to reverse. The prolong state of anxious feeling will be adopted by the individual and form anxious personality (Miu et al., 2009; Miclea, Albu \& Ciuca, 2009). In addition, trait anxiety is much more impactful for children compared to state anxiety as this inborn anxious personality shaped the first default biological vulnerability to fear.

On the other hand, with the advancement of neuroimaging, biological aspects of brain mechanism like brain electrical activity and associated brain part for anxiety helped in understanding the effect of brain mechanism on anxiety which can help in early recognition and intervention. Particularly, neural signatures from brain electrical activity can be detected at the prefrontal cortex and temporal lobe of brain when there is fear response. The evoked fear and anxious feeling mainly due to the activity of prefrontal cortex and temporal lobe. Amygdala and hippocampus which are situated at the temporal lobe responsible for the processing of anxious feeling and interrelate with prefrontal cortex for the interpretation of the feeling when subjects are on task (Antoniadis \& McDonald, 2001). Consequently, brain electrical activity mapping (BEAM) is one of the techniques to identify the activation of specific brain parts that are responsible for fear and anxious feelings.

However, the rising global anxiety prevalence rate remains unresolved. Besides, the current research pool showed limited concern on the specific biological attributed risk factors of brain mechanism on trait anxiety among children and the associated brain locations are still, remained unidentified. So, this research intended to identify the associated brain location for trait anxiety among anxious children. Specifically, this research aimed to study the relationship between trait anxiety and brain locations and also predict the associated brain locations for trait anxiety among anxious children. By identifying the most preliminary factor of brain mechanism, early recognition of inborn trait anxiety personality can help in preventing the early onset of anxiety disorders. This research procedure has undergone an ethical clearance process under JKEUPM (Ethic Committee for Research Involving Human Subject) (JKEUPM-2019-277). Besides, this research is under Geran Putra-IPS, University Putra Malaysia (9677000).

\section{Literature Review}

Trait anxiety is the tendency to experience state anxiety when individuals respond to the perceived threat (Elwood, Wolitzky-Taylor, \& Olatunji, 2012). Individuals who display trait anxiety tend to have a belief that reflects their perception of specific external stimuli and situations as risky or threatening (Spielberger, 1966). Those with a high level of trait anxiety experience a greater intensity of state anxiety to a broader range of situations and objects than most people do, which is why trait anxiety is relatively stable and more like a personality characteristic, instead of a temporary feeling (Miu, Heilman, \& Miclea, 2009; Gros et al., 2007).

GATR Global J. Bus. Soc. Sci. Review 8 (4) $246-259$ (2020) 
Barlow (2002) introduced the triple vulnerability theory, an integrated model combining biological contribution, psychological contribution, and social contribution, in order to explain anxiety disorders in a holistic way. The first vulnerability, or diathesis, is called the generalized biological vulnerability, which explains that anxious genes runs in family through inheritance and the second vulnerability is called the generalized psychological vulnerability, which correlates childhood experiences with anxiety.

Past researches showed that Alteration of dorsolateral prefrontal cortex activity played a role in processing different types of worry and anxious feelings. In fact, different forms of anxiety alter a different hemisphere of the brain that is responsible for anxiety. For instance, patients with elevated trait anxiety, obsessive-compulsive symptoms, and those with an OCD diagnosis were expected to exhibit more right-than-left frontal activity (Smith, Zambrano-Vazquez \& Allen, 2016). Meanwhile, for those who showed social anxiety disorder symptoms, there was an increased in hippocampal and amygdala activity on the left lateralization; for those with generalized anxiety disorders symptoms, there was an elevated right amygdala response (Martin, Ressler, Binder, \& Nemeroff, 2009)

\section{Materials and Method}

This research adopted a cross-sectional research design that involved survey method and neuro-imaging of Quantitative Electroencephalogram (qEEG) brain mapping. Particularly, participants were recruited through online poster advertisements and underwent the first phase of screening phase with the administration of StateTrait Anxiety Inventory for Children-Trait Scale (STAIC-T). Participants were then recruited through purposive sampling and proceed with the second phase of qEEG brain mapping. Data collection processes were conducted in New Mind Brain Health Centre, Life Care Diagnostic and Medical Centre.

\subsection{Participants}

A total of 212 Chinese children in the Kuala Lumpur areas, Malaysia were recruited as participants in this research. Initially, there were a total of 302 participants who agreed to participate in the screening phase. After the screening phase, 80 of them who did not meet the inclusive criteria were excluded from this research and throughout the process, 10 participants decided to withdraw from the research. Participants' age ranged from eight to 13 with mean age, $\mathrm{M}=10.15$, $(\mathrm{SD}=1.65)$ that indicated childhood stage, whereby age eight is the minimal age group as STAIC-T was designed for children with age eight and above based on their understanding level, while age 13 is the cut-off point for childhood stage as suggested by Ministry of Health, Malaysia.

\subsection{Instruments}

In order to achieve the objectives in this research, three instruments were used. Firstly, STAIC-T and Demographic Questionnaire was administered at the first phase as a screening tool to recruit children with score 35 and above as an indication of high trait anxiety and meanwhile, the trait anxiety score of participants were obtained. Secondly, qEEG brain mapping was administered to study the associated brain locations for trait anxiety.

Demographic Questionnaire was developed by researcher in order to obtain the demographic background of participants. This self-report Demographic Questionnaire consisted of five sections, which are gender, age, number of siblings, type of schooling and household monthly income. Demographic Questionnaire was presented in two languages of English and Mandarine to improve the understanding levels of participants.

State-Trait Anxiety Inventory for Children (STAIC) was developed by Charles Spielberger by 1973 in collaboration with Edwards, Lushene, Montuori and Denna Platzek. STAIC consisted of two subscales of State scale and Trait scale with 20 items each. In this research, only Trait scale was adopted to study the trait anxiety score of participants as an indication of biological attributed trait anxious personality. STAIC-T was also

GATR Global J. Bus. Soc. Sci. Review 8 (4) 246 -259 (2020) 
presented in two languages of English and Mandarin so that participants can have better understanding regarding each item. On top of that, STAIC is an ideal instrument to be used as it is a reliable and valid selfreport instrument with 3-points Likert scale (1- Hardly ever; 2- Sometimes; 3-Often) for children aged 8 and above. Spielberger et al. (1983) reported STAIC showed .90 to .93 alpha coefficients for internal consistency and .73 to .86 for test-retest reliability. Besides, both scales showed convergent validity whereby both scales were statistically correlated significantly with other anxiety scales. In addition, both scales in STAIC revealed good internal consistency and test-retest reliability in children population which suitable for current studied participants (Turgeon \& Chartrand, 2003; Spielberger, Gorsuch, \& Lushene, 1970; Hashim, Wan Hasyila, Ang, Azlan Helmy, \& Husyairi, 2018). Besides, Mandarin version of STAIC-T showed good internal consistency of $r=0.83$. The psychometric properties of this short form have been empirically tested, showing adequate internal consistency reliability, good concurrent validity, and excellent construct validity for Chinese population among children (Peng, Johanson \& Chang, 2012; Shek, 1991).

Besides, a Trainers' qEEG brain mapping machine was used to administer brain electrical activity mapping on children participants in order to identify the lighted brain locations during on task state. The Trainers' qEEG brain mapping machine included a Q-Wiz operation device and a cap with 21 electrodes (Fpz, Afz Fp1, Fp2, F7, F8, F3, F4, Fz, T3 T4, T5, T6, C3, C4, Cz, P3, P4 Pz, Oz, O1, and O2) based on International 10-20 system (American Electroencephalographic Society, 1991). qEEG brain mapping techniques had been widely used among the child population (Chabot, Coben, Hirshberg, \& Cantor, 2015; Azouz, Khalil, \& Abdeldayem, 2018). . It is safe, painless and non-invasive to the studied subject (Minho, Byung \& Sungho, 2015; Hackett, 2018). Besides, this is a reliable instrument with alpha coefficient of more than .90 reliability and high test retest reliability over many days and weeks (Chiarenza, 2016). Furthermore, qEEG brain mapping is a valid instrument whereby it showed predictive validity with accurate predictions of outcome and performance on neuropsychological tests. qEEG brain mapping is also well-established by significant and replicable correlations with clinical measures (Thatcher, 2010).

\subsection{Procedures}

At the beginning stage, an online poster was advertised on social media to recruit respondents who were interested in this research. Informed consent form and a short explanation were given on the objectives, procedures and potential risks of this research to both children and parents. Upon signing the informed consent form, STAIC-T was administered to children which took approximately 15 minutes. After that, scores of STAIC-T were calculated and for those with score 35 and above were recruited to follow the second phase of qEEG brain mapping.

During the second phase of qEEG brain mapping, a cap was worn on the participant head followed by the injecting of conductive gel on scalps. After that, participants are required to follow five groups of tasks, a total of 15 tasks as follow:

Task 1: Eyes close, Eyes open, Movie Watching task (Watch a video)

Task 2: Eyes close, Eyes open, Memory task (Memorize 5 digits of number)

Task 3: Eyes close, Eyes open, Imagination task (Imagine the things that they like)

Task 4: Eyes close, Eyes open, Book reading task (Read a story book in mind)

Task 5: Eyes close, Eyes open, Pattern recognizing task (Paring of picture and shadow)

qEEG brain mapping took approximately 40 minutes of administration time. However, for those who are not able to finish all tasks in the scheduled time, they were rescheduled to another date and time to continue with the qEEG brain mapping. Lastly, a stress ball was given to each participant as a token of appreciation. Debrief sessions were conducted so that participants can have better understanding regarding this research. 


\subsection{Data Analysis}

Collected data was analyzed by using IBM Version 2.0 Statistical Package for the Social Sciences (SPSS). For trait anxiety score, the scores from all 20 items in STAIC-T were summed up. A total score of STAIC-T indicated the level of trait anxiety of children participants whereby the higher the score, the more anxious the participants are.

For qEEG brain mapping, a brain map was generated along with a computer-generated report which can aid in the identification and interpretation of brain location. On task brain map for brainwaves with $23-38 \mathrm{~Hz}$ at the Prefrontal Cortex area (Fp1, Fp2, F7, F8, F3, F4) and Temporal lobe (T3, T4) were studied to identify the associated brain part for trait anxiety as shown in Figure 1 with red color as an indication. Furthermore, activation of each brain locations was assessed by intensity of color from $0 \%$ to $30 \%$ whereby $0 \%, 5 \%, 10 \%$, $15 \%, 20 \%, 25 \%$ and $30 \%$ was coded as score $0,1,2,3,4,5$ and 6 respectively in SPSS system as shown in Figure 2. Subsequently, the score for each brain locations were summed up that indicated the intensity of activation whereby the higher the score, the higher the intensity of activation of the particular brain location when on task which resulted from stronger trait anxiety.

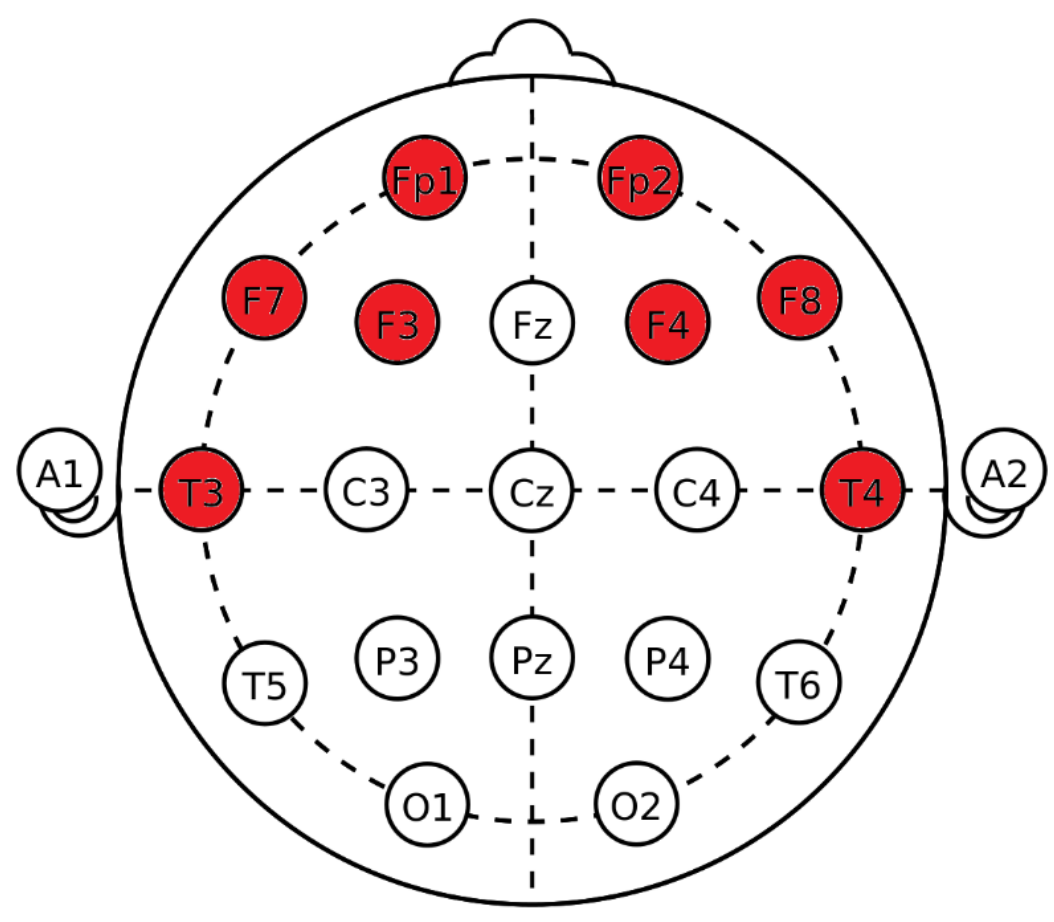

Figure 1. Studied brain locations according to the International 10-20 System. 


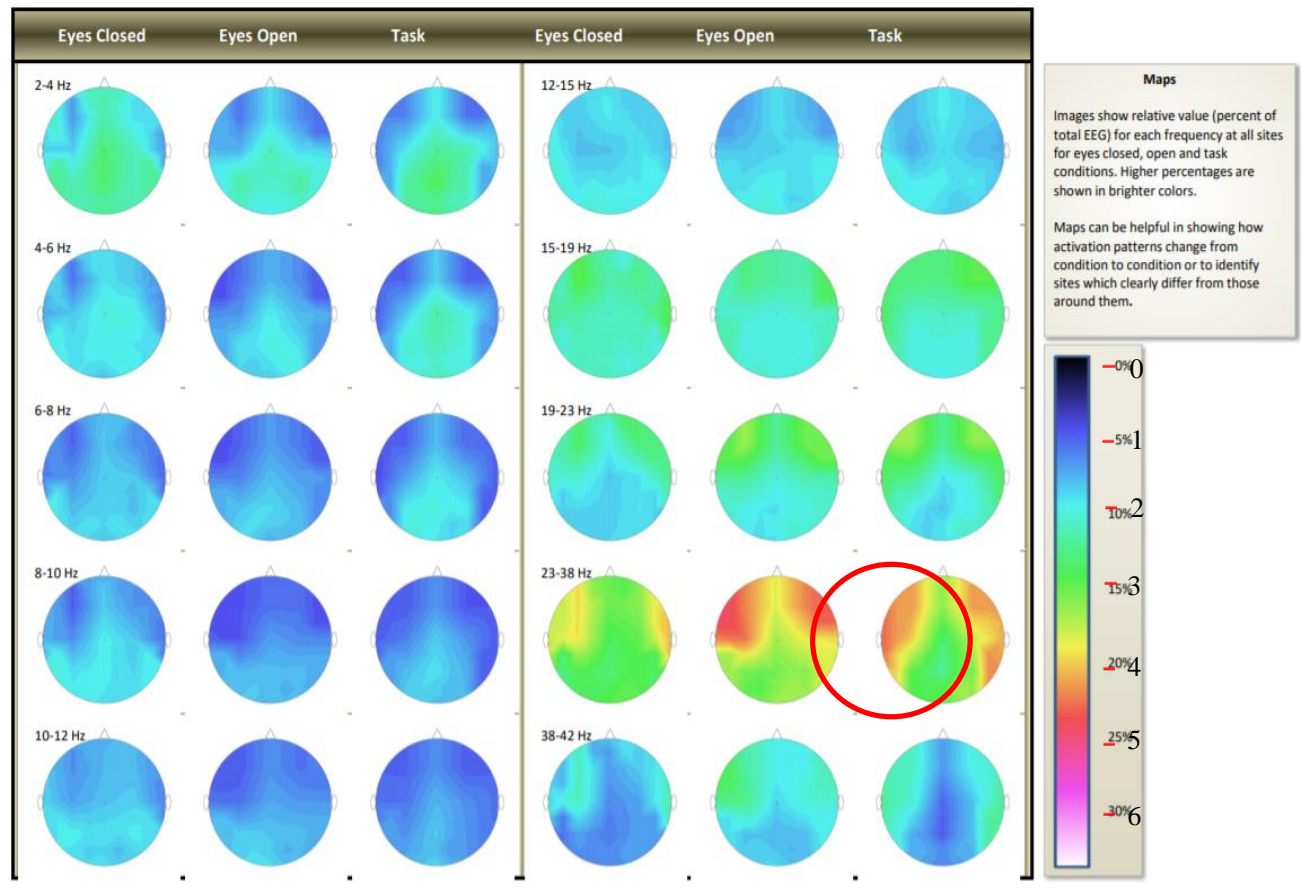

Figure 2. Lighted brain locations and coding system for brain electrical activity mapping.

After that, descriptive analysis was carried out followed by inferential analysis. Particularly, Pearson's Correlation was carried out to study the relationship between trait anxiety and all brain locations among anxious children. Furthermore, Step-wise Multiple Regression was carried out to predict the associated brain locations for trait anxiety among children.

\section{Results}

This section discussed the analyzed results from SPSS. For the first section, discussion on the frequency and percentage distribution of participants' demographic profile were revealed in Table 1 followed by descriptive analysis on trait anxiety level. It is then followed by the discussion on the results for brain electrical activity mapping through inferential analysis.

\subsection{Descriptive analysis for Demographic Profile}

Descriptive analysis was carried out to understand the demographic profile of participants. Demographic profile of respondents was obtained through Demographic Questionnaire in terms of gender, number of siblings, type of schooling and monthly household income. Table 1 presented the distribution of demographic profiles for 212 participants

Table 1. Frequency and percentage distribution for demographic profile.

\begin{tabular}{ccc}
\hline Variable & Frequency (n) & Percentage (\%) \\
\hline Gender & 106 & 50.00 \\
Male & 106 & 50.00
\end{tabular}

Female 


\section{Number of Siblings}

1

2

3

4

5

$>5$

Type of Schooling

Public School
Private School

International School

Household Monthly Income

\begin{tabular}{ccc} 
Household Monthly Income & & \\
T20 & 38 & 17.92 \\
M40 & 135 & 63.68 \\
B40 & 39 & 18.40 \\
\hline Total & $\mathbf{2 1 2}$ & $\mathbf{1 0 0 . 0 0}$ \\
\hline
\end{tabular}

25.5

38.2

23.60

9.40

2.80

0.50

72.60

3.80

$50 \quad 23.60$

154$$
60
$$

(60)




\subsection{Descriptive Analysis for Trait Anxiety}

Trait anxiety level for children participants were assessed through the administration of STAIC-T. Based on Figure 3, the mean score for STAIC-T among studied participants is $\mathrm{M}=45.84$ ( $\mathrm{SD}=5.82$ ) whereby the lowest cut-off point is 35 and 58 as the highest point. Particularly, score 50 has the higher frequency of 32 participants among other scores. On the contrary, the highest score of 58 showed the least frequency of only one person. Overall, most of the studied participants in the Kuala Lumpur area are prone to exhibit higher levels of trait anxiety.

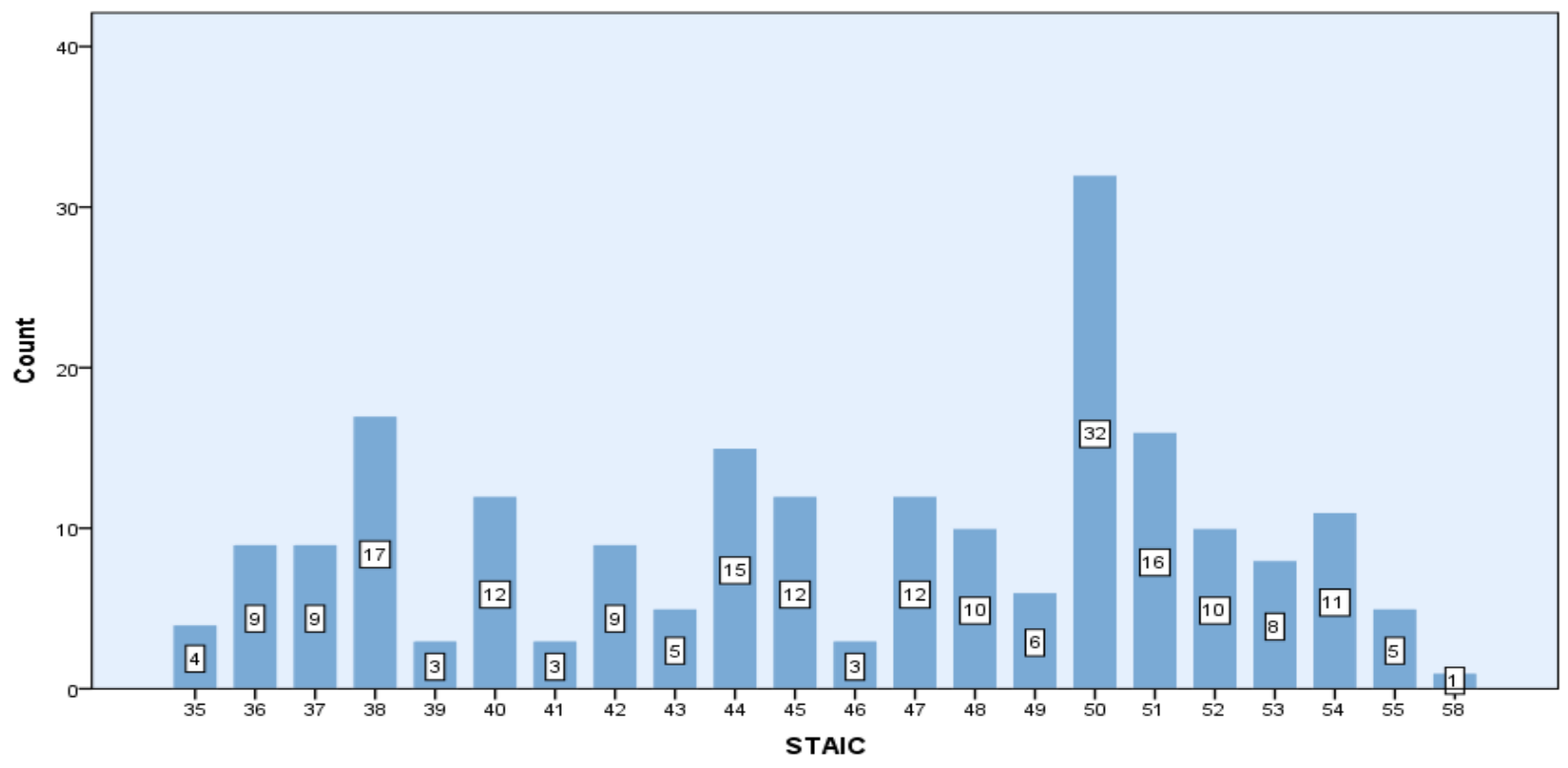

Figure 3. Distribution of trait anxiety level.

On top of that, there is an obvious difference in the distribution of trait anxiety level when comparing among gender as shown in Figure 4. In fact, male has the mean trait anxiety score of $\mathrm{M}=41.45,(\mathrm{SD}=4.30)$ and female has the mean score of $\mathrm{M}=50.24$, $(\mathrm{SD}=3.25)$. Based on Figure 4, females showed higher level of trait anxiety with minimal score of 40 and highest score of 58 while males showed lower trait anxiety level with minimal score of 35 and highest score of 52. Overall, female children participants exhibit higher levels of trait anxiety compared to male children participants. 


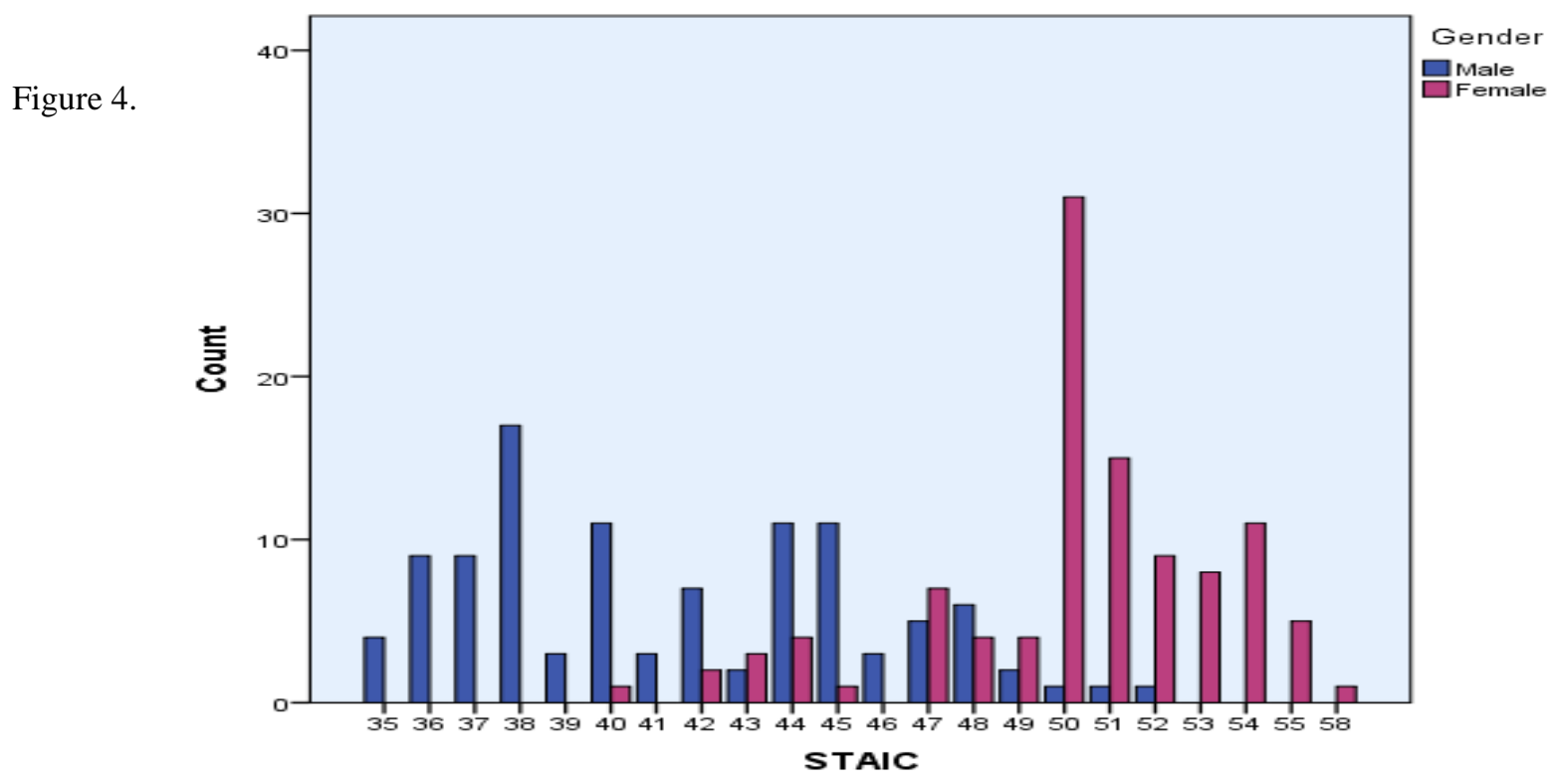

Distribution of trait anxiety level for male and female.

\subsection{Inferential Analysis for Brain Locations}

In order to achieve the proposed objective, inferential analysis was carried out to study the brain locations obtained through qEEG brain mapping. For instance, Pearson's Correlation was carried out to study the relationship between trait anxiety and the 8 studied brain locations that located at Prefrontal Cortex area (Fp1, Fp2, F7, F8, F3, F4) and Temporal Lobe (T4, T4). Based on Table 5, results showed that there is a significant relationship between trait anxiety and all the eight brain locations among anxious children as shown in Table 2. Particularly, children' trait anxiety showed a negative significant relationship with brain location Fp1 of $r$ $(210)=-.184, \mathrm{n}=212, \mathrm{p}<0.01$. Besides, children' trait anxiety showed positive significant relationships with seven brain locations of Fp2, F3, F4, F7, F8, T3 and T4. Specifically, Fp2 has a value of r (210) =.195, n = $212, \mathrm{p}<0.01, \mathrm{~F} 3$ has a value of $\mathrm{r}(210)=.149, \mathrm{n}=212, \mathrm{p}<0.05, \mathrm{~F} 4$ has a value of $\mathrm{r}(210)=.198, \mathrm{n}=212, \mathrm{p}$ $<0.01, \mathrm{~F} 7$ has a value of $\mathrm{r}(210)=.154, \mathrm{n}=212, \mathrm{p}<0.05, \mathrm{~F} 8$ has a value of $\mathrm{r}(210)=.349, \mathrm{n}=212, \mathrm{p}<0.01$, $\mathrm{T} 3$ has a value of $\mathrm{r}(210)=.136, \mathrm{n}=212, \mathrm{p}<0.05$ and $\mathrm{T} 4$ has a value of $\mathrm{r}(210)=.143, \mathrm{n}=212, \mathrm{p}<0.05$.

Table 2. Pearson's Correlation (r) of children' trait anxiety level with brain locations.

\begin{tabular}{|c|c|c|c|c|c|c|c|c|c|c|}
\hline & Variables & 1 & 2 & 3 & 4 & 5 & 6 & 7 & 8 & 9 \\
\hline 1. & a STAIC & 1 & & & & & & & & \\
\hline 2. & Fp1 & $-.184 * *$ & 1 & & & & & & & \\
\hline 3. & Fp2 & $.195^{* *}$ & -.110 & 1 & & & & & & \\
\hline 4. & F3 & $.149^{*}$ & -.066 & -.020 & 1 & & & & & \\
\hline 5. & F4 & $.198 * *$ & -.021 & .108 & $.146^{*}$ & 1 & & & & \\
\hline 6. & F7 & $.154^{*}$ & -.055 & .051 & $.936 * *$ & .108 & 1 & & & \\
\hline 7. & F8 & $.349 * *$ & -.107 & .097 & .121 & .125 & $.177 * *$ & 1 & & \\
\hline
\end{tabular}




\begin{tabular}{rrrrrrrrrrr}
\hline 8. & $\mathbf{T 3}$ & $.136^{*}$ & -.087 & .046 & $.914 * *$ & $.135^{*}$ & $.934 * *$ & $.134 *$ & 1 \\
9. & $\mathbf{T 4}$ & $.143^{*}$ & -.049 & .044 & $.877 * *$ & .078 & $.925 * *$ & $.180 * *$ & $.904 * *$ & 1 \\
\hline
\end{tabular}

Notes. * significant level at $\mathrm{p}<0.05 ., * *$ significant level at $\mathrm{p}<0.01$; aSTAIC-T State-Trait Anxiety Inventory for Children

Consequently, Step-wise Multiple Regression was carried out to predict the associated brain locations for trait anxiety. Based on Table 3, out of the eight brain locations, four brain locations of F8, Fp2, F4 and Fp1 are significant predictors for trait anxiety among anxious children with significant regression equation of $(\mathrm{F}(4,207)$ $=11.49, \mathrm{p}<0.01)$. Model 4 of Step-wise Multiple Regression showed that studied participants predicted trait anxiety $=42.02+(2.06) \mathrm{F} 8+(1.02) \mathrm{Fp} 2+(1.04) \mathrm{F} 4-(.94)$ Fp1. Furthermore, Model 4 from Step-wise Multiple Regression showed an R2 of 0.18 indicated that these four brain locations of F8, Fp2, F4 and Fp1 contributed $18 \%$ to trait anxiety among anxious children. Besides, as an indication from value VIF $<10$ for all four predictors for trait anxiety, there is no multicollinearity presented.

Table 3. Step-wise Multiple Regression predicting trait anxiety from brain locations

\begin{tabular}{|c|c|c|c|c|c|}
\hline Model & R2 & Adjusted R2 & $\mathrm{F}$ & $\beta$ & $\mathrm{p}$ \\
\hline Model 1 & .12 & .12 & 29.05 & & \\
\hline Constant & & & & 42.99 & \\
\hline F8 & & & & 2.37 & .000 \\
\hline Model 2 & .15 & .14 & 17.98 & & \\
\hline Constant & & & & 41.87 & \\
\hline F8 & & & & 2.25 & .000 \\
\hline Fp2 & & & & 1.25 & .014 \\
\hline Model 3 & .17 & .15 & 13.78 & & \\
\hline Constant & & & & 41.00 & \\
\hline F8 & & & & 2.15 & .000 \\
\hline Fp2 & & & & 1.12 & .027 \\
\hline F4 & & & & 1.07 & .030 \\
\hline Model 4 & .18 & .16 & 11.49 & & \\
\hline Constant & & & & 42.02 & \\
\hline F8 & & & & 2.06 & .000 \\
\hline
\end{tabular}

GATR Global J. Bus. Soc. Sci. Review 8 (4) $246-259$ (2020) 


\begin{tabular}{ccc}
\hline Fp2 & 1.02 & .042 \\
F4 & 1.04 & .034 \\
Fp1 & -0.94 & .047 \\
\hline
\end{tabular}

\section{Discussion}

This section covered the discussion on previous results on brain electrical activity mapping. Next, implications of study were revealed. Besides, the potential limitations in this study were discussed along with some recommendations for future study. Referring to the results on the relationship between trait anxiety and brain locations, results showed that there is a significant relationship between all the eight brain locations of Fp1, Fp2, F7, F8, F3, F4, T3 and T4. Specifically, brain location Fp1 showed a negative significant relationship with trait anxiety among children in which when Fp1 area is less activated, the child is more likely to exhibit higher trait anxiety and vice versa. As for other brain locations of Fp1, F7, F8, F3, F4, T3 and T4, a positive significant indicated that the more activated the brain locations, is more likely for the child to exhibit trait anxiety and vice versa. These research findings again tally with previous findings on the association of prefrontal cortex, amygdala and hippocampus with fear responses (Antoniadis \& McDonald, 2001). Fear responses that create anxious feelings are the results from the activation of the prefrontal cortex along the amygdala and hippocampus in temporal lobe. By detecting the activation of brain electrical activity through mapping, default current anxious brain state of children can be identified.

Subsequently, results from Stepwise Multiple Regression showed that brain locations F8, Fp2, F4 and Fp1 are the significant predictor for trait anxiety among children. Research findings showed that all the four significant predictors are from prefrontal cortex area while brain locations, T3 T4 of temporal lobe were eliminated. It showed that trait anxiety among children is more associated with the activation of prefrontal cortex rather than temporal lobe. For instance, this finding indicated that trait anxiety among children is associated with the activation of prefrontal cortex rather than the activity from amygdala and hippocampus which are situated at the temporal lobe. However, this finding differs from the findings presented by previous study on fear expression during fear acquisition whereby anxiety is associated with increased amygdala activation (Lissek et al., 2005; Indovina et al., 2011). This discrepancy could be attributed to the faster growing speed of frontal lobe for children. During childhood stage, frontal lobe of children are more active compare to other lobe of brain that associated with developmental ability of focus attention, reasoning and self-control. This is caused by the significant changes in neural functioning account for cognitive advances in childhood which resulted in more activated prefrontal cortex (Kolb \& Whishaw, 2009; Overman, Bachevalier, Turner, \& Peuster, 1992). Consequently, these four locations act as a biomarker that indicates trait anxiety symptoms among children. It helps in early recognition and identification for anxiety disorders since young in order to administer more targeted intervention.

By studying the brain electrical activity of children with trait anxiety, research findings can help in enriching the current research pool on related psychology fields. Research findings on associated brain locations provide some basic information for researchers on the biological aspect of risk factor for anxiety among children which can help in early recognition of anxiety disorders. Furthermore, by identifying the associated brain locations as predictors for trait anxiety, more targeted treatment plans can be implemented to children who are born with this biological vulnerability. In fact, the early intervention plan will be much more effective as the proposed intervention can target the biological brain mechanism since young. However, this research has some potential limitations. Firstly, this research only focused on a specific group of Chinese children in the Kuala Lumpur area. For instance, the generalizability and representativeness of the research findings might be affected. 
Subsequently, this research restricted the age range from eight to 13 years old which may not represent the whole childhood stage. Besides, the definition and cut-off age for the childhood stage may vary with different cultural background and country, so the proposed age in this research may not be able to represent the exact childhood stage. Next, in terms of brain electrical activity mapping, research findings only focused on the activation of brain locations during on task state of participants which limited the research findings on eyes closed state and eyes open state.

\section{Conclusion}

In conclusion, biological brain mechanism played a crucial role in manipulating the level of trait anxiety of children since birth. By studying the specific associated brain locations for certain disorders. Researchers and practitioners in related fields can target one of the prominent risk factors of anxiety among children. As for trait anxiety among children, the identified associated brain locations for trait anxiety can help in early recognition of anxiety disorders and administration of early interventions which target their biological brain directly. By addressing the biological risk factors, children are more resilient to cope with stress and reach their developmental milestones at a healthy pace. In order to reduce the impact of limitations, some recommendations will be discussed in order to improve the future study. Firstly, future study should revise the sampling method and selection research participants to improve the generalizability and representativeness of the study (Kukull, \& Ganguli, 2012). Furthermore, sample diversity should be taken into consideration when studying children of a certain developmental age. Lastly, future studies can intensify the findings of brain electrical mapping on trait anxiety by incorporating the other two phases of eyes opening and eyes closing in data analysis.

\section{Acknowledgement}

First of all, I am really grateful to God for the blessings that have been given to me and I am able to complete this research on "Brain Electrical Activity Mapping (BEAM) on Trait Anxiety among Anxious Chinese Children".

Next, I would like to express my deepest gratitude and appreciation to my research supervisor, Dr. Asmidawati binti Ashari, who has provided invaluable suggestions, guides and comments throughout the research. Besides, special thanks to all my co-supervisor Dr Rahimah and Dr Wan Aliaa who showed patience to me and motivated me to overcome any challenges in completing this research. Lastly, thanks to my site supervisor, Hiro Koo Kian Yong who supervised me in administering qEEG brain mapping.

Millions of appreciations to New Mind Brain Health Centre for approving my application to carry out the data collection process there. Thanks for providing a good and well-equipped environment for me to collect my data. Special thanks to all the staff there who had helped me in this period so that I can complete my data collection smoothly.

Besides, I am really grateful to have my beloved family and friends who are always by my side and support me. I dedicate this thesis to my parents, siblings and loved ones. I would also like to say big thanks to my fellow course mate, Tun Ju Ern who had gone through the journey of completing this research with me.

On top of that, million thanks to Geran Putra-IPS, University Putra Malaysia for funding me to conduct this research. Those funds lighten my financial burden to prepare all the research materials and conduct this research.

Last words may God bless all of them and indeed, this research provided insight for me as a master student and also as a human being. Thank you very much. 


\section{References}

Nomenclature, S. E. P. (1991). American electroencephalographic society guidelines for. Journal of clinical Neurophysiology, 8(2), 200-2. https://10.1097/00004691-199104000-00007

Antoniadis, E. A., \& McDonald, R. J. (2001). Amygdala, hippocampus, and unconditioned fear. Experimental Brain Research, 138(2), 200-209. https://doi.org/10.1007/s002210000645

Averill, J. R. (1976). Emotion and anxiety: Sociocultural, biological, and psychological determinants.

Azouz, H. G., Khalil, M., \& Abdeldayem, S. H. (2018). Quantitative electroencephalographic changes in children with autism spectrum disorders. Alexandria Journal of Pediatrics, 31(3), 97. https://10.4103/AJOP.AJOP_29_18

Barlow, D. H. (2004). Anxiety and its disorders: The nature and treatment of anxiety and panic. Guilford press.

Bittner, A., Egger, H. L., Erkanli, A., Jane Costello, E., Foley, D. L., \& Angold, A. (2007). What do childhood anxiety disorders predict?. Journal of Child Psychology and Psychiatry, 48(12), 1174-1183. https://doi.org/10.1111/j.14697610.2007.01812.x

Chabot, R. J., Coben, R., Hirshberg, L., \& Cantor, D. S. (2015). QEEG and VARETA based neurophysiological indices of brain dysfunction in attention deficit and autistic spectrum disorder. Austin J Autism \& Relat Disabil, 1(2), 1007. DOI 10.1007/978-1-4614-6843-1_6

Chiarenza, G. A., Chabot, R., Isenhart, R., Montaldi, L., Chiarenza, M. P., Torto, M. G. L., \& Prichep, L. S. (2016). The quantified EEG characteristics of responders and non-responders to long-term treatment with atomoxetine in children with attention deficit hyperactivity disorders. International Journal of Psychophysiology, 104, 44-52. https://doi.org/10.1016/j.ijpsycho.2016.04.004

Elwood, L. S., Wolitzky-Taylor, K., \& Olatunji, B. O. (2012). Measurement of anxious traits: a contemporary review and synthesis. Anxiety, Stress \& Coping, 25(6), 647-666. https://doi.org/10.1080/10615806.2011.582949

Engel, G. L. (1997). From biomedical to biopsychosocial: Being scientific in the human domain. Psychosomatics, 38(6), 521-528. https://doi.org/10.1016/S0033-3182(97)71396-3

Hashim, E., Hasyila, W. W., Ang, Y., Helmy, A. A., \& Husyairi, H. (2018). Psychometric properties of the Malay Translated Spielberger State-Trait Anxiety Inventory in exploring parental anxiety. Med Health, 13, 106-16. https://doi.org/10.17576/MH.2018.130.11

Indovina, I., Robbins, T. W., Núñez-Elizalde, A. O., Dunn, B. D., \& Bishop, S. J. (2011). Fear-conditioning mechanisms associated with trait vulnerability to anxiety in humans. Neuron, 69(3), 563-571.

https://doi.org/10.1016/j.neuron.2010.12.034

Kolb, B., \& Whishaw, I. Q. (2009). Fundamentals of human neuropsychology. New York, NY: Worth.

Kukull, W. A., \& Ganguli, M. (2012). Generalizability: the trees, the forest, and the low-hanging fruit. Neurology, 78(23), 1886-1891. https://10.1212/WNL.0b013e318258f812

Lissek, S., Powers, A. S., McClure, E. B., Phelps, E. A., Woldehawariat, G., Grillon, C., \& Pine, D. S. (2005). Classical fear conditioning in the anxiety disorders: a meta-analysis. Behaviour research and therapy, 43(11), 1391-1424. https://10.1016/j.brat.2004.10.007

Cooper, M., Vafadari, K., \& Hieda, M. (2015). Current issues and emerging trends in medical tourism. New York: IGI Global. DOI: 10.4018/978-1-4666-8574-1

Martin, E. I., Ressler, K. J., Binder, E., \& Nemeroff, C. B. (2009). The neurobiology of anxiety disorders: brain imaging, genetics, and psychoneuroendocrinology. Psychiatric Clinics, 32(3), 549-575. doi: https://10.1016/j.psc.2009.05.004

Ciuca, A., Albu, M., Perte, A., Budau, O., Miclea, S., \& Miclea, M. (2011). The Romanian adaptation of the psychiatric diagnostic screening questionnaire (PDSQ). Cognition, Brain, Behavior, 15(1), 69.

Kim, M., Kim, B. H., \& Jo, S. (2014). Quantitative evaluation of a low-cost noninvasive hybrid interface based on EEG and eye movement. IEEE Transactions on Neural Systems and Rehabilitation Engineering, 23(2), 159-168. https://10.1109/TNSRE.2014.2365834

Miu, A. C., Heilman, R. M., \& Miclea, M. (2009). Reduced heart rate variability and vagal tone in anxiety: trait versus state, and the effects of autogenic training. Autonomic Neuroscience, 145(1-2), 99-103. https://doi.org/10.1016/j.autneu.2008.11.010

Overman, W., Bachevalier, J., Turner, M., \& Peuster, A. (1992). Object recognition versus object discrimination: Comparison between human infants and infant monkeys. Behavioral Neuroscience, 106(1), 15. https://doi.org/10.1037/0735-7044.106.1.15 
Peng, H. H., Johanson, R. E., \& Chang, M. H. (2012). Career indecision and state anxiety of returned international Chinese undergraduate students in Taiwan. International Journal of Psychology and Counselling, 4(9), 106-114. https://10.5897/IJPC12.003

Pine, D. S., Cohen, P., Gurley, D., Brook, J., \& Ma, Y. (1998). The risk for early-adulthood anxiety and depressive disorders in adolescents with anxiety and depressive disorders. Archives of general psychiatry, 55(1), 56-64. doi:10.1001/archpsyc.55.1.56

Department of Statistics Malaysia. (2015). Report of household income and basic amenities survey 2014.

Shek, D. T. (1991). The factorial structure of the Chinese version of the State-Trait Anxiety Inventory: A confirmatory factor analysis. Educational and Psychological Measurement, 51(4), 985-997. doi: https://doi.org/10.1177/001316449105100418

Smith, E. E., Zambrano-Vazquez, L., \& Allen, J. J. (2016). Patterns of alpha asymmetry in those with elevated worry, trait anxiety, and obsessive-compulsive symptoms: A test of the worry and avoidance models of alpha asymmetry. Neuropsychologia, 85, 118-126. https://doi.org/10.1016/j.neuropsychologia.2016.03.010

Spielberger, C. D., Gorsuch, R. L., \& Lushene, R. E. (1970). Manual for the state-trait anxiety inventory. Palo Alto, CA: Consulting Psychologists Press.

Spielberger, C. D., Gorsuch, R. L., Lushene, R., Vagg, P. R., \& Jacobs, G. A. (1983). Manual for the state-trait anxiety inventory (Palo Alto, CA, Consulting Psychologists Press). Inc.

Thatcher, R. W. (2010). Validity and reliability of quantitative electroencephalography. Journal of Neurotherapy, 14(2), 122-152. https://10.1080/10874201003773500

Turgeon, L., \& Chartrand, É. (2003). Psychometric properties of the French-Canadian version of the state-trait anxiety inventory for children. Educational and Psychological Measurement, 63(1), 174-185. https://doi.org/10.1177/0013164402239324

Woodward, L. J., \& Fergusson, D. M. (2001). Life course outcomes of young people with anxiety disorders in adolescence. Journal of the American Academy of Child \& Adolescent Psychiatry, 40(9), 1086-1093. https://10.1097/00004583-200109000-00018 